\title{
Formación de traductores universitarios: experiencias interdisciplinarias en el campo de la traducción de las ciencias sociales y humanas ${ }^{1}$
}

\author{
Beatriz Emilce Cagnolati \\ bcagnolati@fahce.unlp.edu.ar \\ https://orcid.org/0000-0003-0626-1183 \\ Universidad Nacional de La Plata, Argentina
}

\section{Resumen}

Este trabajo se inscribe en la formación universitaria de traductores, en el par de lenguas francés/ español, y se fundamenta en nuestra experiencia de docencia e investigación. Presenta los contenidos del último nivel de traducción científico-técnica, focalizando en las actividades teórico-prácticas de la etapa final de la carrera, en particular los trabajos interdisciplinarios de traducción de textos de ciencias sociales y humanas, con la participación del alumnado, la docente investigadora de sociología y economía del trabajo (especialista de referencia) y la docente de la cátedra (docente coordinadora). Los objetivos de este artículo son, por un lado, difundir esas experiencias por medio del comentario de los informes de traducción elaborados por las alumnas intervinientes, quienes identifican y ejemplifican los problemas de traducción de los artículos científicos en el área sociológica, aportan las soluciones mediante las estrategias puestas en juego y fundamentan el proceso traductivo con base en los principios teóricos que han incorporado a lo largo de sus estudios; por otro lado, exponer el análisis sobre las anomalías que mostraron las primeras reformulaciones, generalmente como producto del mecanismo de interferencia entre el discurso de partida y la producción discursiva, con incidencia en niveles peritextuales. En un contexto de aula taller, el análisis retrospectivo de las sucesivas producciones desarrolla la autocrítica constructiva y consolida la autonomía, con el objetivo de ampliar la competencia traductora.

Palabras clave: ciencias sociales y humanas; formación en traducción en Argentina; traducción francés/español.

Translator Education at the University-Level: Interdisciplinary Experiences in the Field of Social and Human Sciences Translation

\footnotetext{
Abstract

This article addresses translator's university education in the French-Spanish pair of languages, based on the author's teaching and research experience. The article examines the contents of senior

1 Este artículo está relacionado parcialmente con la tesis doctoral "La producción discursiva en la traducción de textos pragmáticos francés/castellano: estudio traductológico a través de los enunciados elípticos con anomalías", defendida en noviembre del 2010 (Cagnolati, 2010).
} 
level scientific-technical translation assignments, focusing in theoretical-practical activities at the last stage of the curriculum, specifically in interdisciplinary translation works of social and human science texts. The study involved the participation of students, the teacher-researcher on social science and labor economics (the expert) and the lecturer (teacher in charge). This article aims to spread these experiences, in two ways: first, by commenting translation reports done by participating students, where they identify and give examples about translation challenges of scientific articles in the field of social science, they provide solutions through the strategies applied and support the translation process based on the theoretical principles they have learned over the course of their studies; second, it intends to present an analysis of the anomalies displayed by earlier reformulations, generally as a result of interference between source and target discourses, which has an impact on peritextual aspects. In the context of a workshop classroom, a retrospective analysis of subsequent productions develops constructive self-criticism and strengthens autonomy, conducive to enhance translator's competence.

Keywords: Human and social sciences; translation training in Argentina; French-Spanish translation.

Formation des traducteurs universitaires : des expériences interdisciplinaires dans le domaine des sciences sociales et humaines

\section{Résumé}

Le présent travail concerne la formation universitaire des traducteurs pour la combinaison français/ espagnol, et repose sur notre expérience dans l'enseignement et la recherche. Il présente les contenus $\mathrm{du}$ dernier niveau de traduction scientifique et technique, et se focalise sur les activités théoriques et pratiques de l'étape finale des études, notamment sur les travaux interdisciplinaires de traduction de textes des sciences sociales et humaines, dans lesquels ont pris part les étudiantes, l'enseignante et chercheure en sociologie et en économie du travail (spécialiste de référence) et l'enseignante du cours (enseignante coordinatrice). Les objectifs de cet article sont, d'une part, diffuser ces expériences par le biais du commentaire des rapports des traductions fournis par les étudiantes, où elles identifient les problèmes de traduction des articles scientifiques du domaine de la sociologie, en donnent des exemples, apportent des solutions à travers des stratégies mises en jeu, et expliquent le processus de traduction sur la base des principes théoriques incorporés au long de leurs études. D'autre part, exposer l'analyse sur les anomalies qui se sont montrées dans les premières reformulations, généralement provoquées par le mécanisme d'interférence du va-et-vient entre le discours de départ et la production discursive, dont les conséquences atteignent des niveaux péritextuels. Dans un contexte d'atelier, l'analyse rétrospective des productions postérieures est censée développer l'autocritique constructive et consolider l'autonomie dans le but d'élargir la compétence de traduction.

Mots-clés : sciences sociales et humaines ; formation en traduction en Argentine ; traduction français/espagnol. 


\section{Introducción}

Dentro de la complejidad que implica el diseño de una formación en Traducción, se hace necesario exponer a los futuros traductores al tratamiento de temas y géneros discursivos variados, con miras a proporcionar las herramientas para ingresar a un mundo laboral en el que conviven producciones textuales científicas, jurídicas, ensayísticas, literarias, audiovisuales, entre otras especializaciones (Hurtado Albir, 1996, p. 31).

Según la norma del Instituto Argentino de Normalización y Certificación (IRAM) 17100 (IRAM, 2019) ${ }^{2}$ sobre servicios de traducción, especialidad es el "área temática, esfera de conocimiento o actividad con su propia cultura, características lingüísticas y contexto social especializados", definición que atañe directamente a la traducción científica, técnica o jurídica, las que a su vez contienen subáreas de conocimiento en permanente cambio y actualización.

De manera indirecta, también la traducción literaria está vinculada con las lenguas especializadas (Malinowski Rubio, 2003). ${ }^{3}$ Con esto

2 La norma IRAM 17100 es una adopción modificada de la norma ISO 17100: 2015 Translation services - Requirements for translation services, y de su enmienda, la ISO 17100:2015/Amd. 1:2017.

3 Por ejemplo, Antoine de Saint-Exupéry, escritor y aviador, obtuvo el premio literario Femina en 1931 con Vol de nuit, una obra que muestra abundante empleo de términos de la aviación, lo que contribuye a la verosimilitud de la ficción. En las primeras páginas de Vol de nuit, el escritor cita referencias geográficas argentinas, compara la inmensidad patagónica con la extensión africana y utiliza términos especializados (radio navigant, postes de la ligne, escales):

'San Julián en vue; nous atterrirons dans quelques minutes.'

Le radio navigant passait la nouvelle à tous les postes de la ligne.

Sur deux mille cinq cents kilomètres, du détroit de Magellan à Buenos Aires, des se señala que los léxicos especializados permean tanto las publicaciones de circulación del conocimiento como las obras literarias. Sin embargo, se ha convenido que los textos especializados están dirigidos a un público formado en un área específica y pertenecen a los lenguajes de especialidad (Hurtado Albir, 2013, p. 59). ${ }^{4}$

El conocimiento se ha clasificado según criterios que agrupan a las ciencias en duras o blandas, fácticas o formales, pero nos interesa consignar que, a nivel local, el Consejo Nacional de Investigaciones Científicas y Técnicas (CONICET) divide el conocimiento en cuatro grandes áreas: ciencias agrarias, ingeniería y de materiales; ciencias biológicas y de la salud; ciencias exactas y naturales, y asigna el cuarto y último lugar a las ciencias sociales y humanidades (CONICET, s. f.). Dentro de esta última categoría, el debate académico clásico sobre la diferenciación entre "ciencias humanas" y "ciencias sociales" según su objeto de estudio - las primeras estudian al ser humano como individuo, mientras que las ciencias sociales lo estudian en relación con el grupo, los sistemas sociales y las relaciones sociales - parecería tender a asimilar ambos conceptos, dado que sus fronteras son permeables (Berrichi, 2012, p. 16).

escales semblables s'échelonnaient ; mais celle-ci s'ouvrait sur les frontières de la nuit comme, en Afrique, sur le mystère, la dernière bourgade soumise (1931, pp. 18-19).

4 Los textos especializados incluyen, entre otros, a los textos científicos y técnicos. Los primeros se proponen difundir universalmente la producción del conocimiento en la comunidad científica, se manifiestan con predominio de secuencias argumentativas y descriptivas; los segundos tienen diversas misiones, entre ellas, anunciar productos empresariales, publicitar su consumo, describir procesos industriales, lo que se expresa, principalmente, por medio de secuencias descriptivas y exhortativas (Gamero Pérez, 2001, pp. 26-29). 
En el terreno de la traducción de las ciencias sociales, Joshua Price (2010) señala que la mayoría de los científicos sociales buscan comunicar universalmente sus textos recurriendo a la lengua como instrumento. En cambio, este autor propone que la traducción contribuye a la clarificación conceptual en permanente movimiento y en diálogo con la investigación de las ciencias sociales, sin necesidad ni conveniencia de establecer una conversación universal (pp. 152, 155, 158). ${ }^{5}$

Michèle Leclerc-Olive da su opinión sobre la traducción de las ciencias sociales, en contraste con la traducción de las ciencias formales y de la literatura:

Si bien conviene reconocer que los escritos de las ciencias humanas se nutren de la lengua común - y como tales su traducción se enmarca en las inquietudes generales de la traductología-, no es menos cierto que la mayor parte de esos escritos tienen una ambición conceptual que el traductor no puede ignorar. Por lo tanto, la traducción de las ciencias humanas no goza de la libertad parcial de la traducción literaria ni del carácter a veces obligatorio de la traducción de las ciencias naturales y de las disciplinas formales, cuando cree que puede apoyarse en la constitución de glosarios (2016, párr. 2; la traducción es nuestra). ${ }^{6}$

5 Price discute las reglas enunciadas por Wallerstein como guía de la labor del traductor de ciencias sociales (2010, p. 154), considerándolas útiles, pero a la vez prescriptivas e insuficientes para generalizar su aplicación al amplio espectro de saberes que comparten los textos de ciencias sociales y sus culturas de escritura (2010, pp. 154-158).

6 «S'il convient de reconnaître aux écrits de sciences humaines leur ancrage dans la langue commune - et à ce titre le fait que leur traduction connaît les inquiétudes générales de la traductologie —, il n'en reste pas moins qu'ils ont pour la plupart une ambition conceptuelle que le traducteur ne peut ignorer. Leur traduction n'a donc ni la liberté partielle de la traduction littéraire ni le caractère parfois obligatoire de la traduction dans les sciences de la nature et les disciplines formelles, lorsqu'elle
Por su parte, Gisèle Sapiro (2012) avanza en identificar al ensayo como el género discursivo privilegiado de circulación de las ciencias humanas y sociales (p. 12), con una escritura menos formalizada que la utilizada por las ciencias naturales y con elementos narrativos propios del lenguaje literario, con la consiguiente complejización de la tarea de traducir (p. 10). La autora propone entonces, por un lado, la especialización de los traductores y, por otro, la formación en traducción en todas las disciplinas de las ciencias humanas como una práctica intelectual y de facilitación de la circulación de las ideas (p. 15).

Ahora bien, el proceso de traducción ha sido estudiado sistemáticamente desde diversos enfoques cognitivos, a partir de la segunda mitad del siglo xx (Hurtado Albir, 2013, pp. 128, 130). Por ejemplo, en el campo de la traducción de textos pragmáticos o vehiculares, Jean Delisle describe el proceso heurístico de la traducción e identifica tres fases: comprensión, reformulación y análisis justificativo (1984, pp. 69-85).

Dentro de la reformulación del léxico, la traducción especializada plantea problemas que se resuelven apelando, entre otros recursos, a diccionarios generales, bases de datos lexicológicos, ${ }^{7}$ diccionarios especializados, bases de datos terminológicos y textos paralelos - es decir, textos de dos culturas diferentes, producidos independientemente y pertenecientes al mismo género o tipo textual, en los que son análogos los factores de las situaciones comunicativas de uso de los dos textos (emisor, tiempo, motivo, destinatarios, medio, función comunicativa,

croit pouvoir s'appuyer sur l'établissement de glossaires » (Leclerc-Olive, 2016, párr. 2).

7 Por ejemplo, el Diccionario de la lengua española de la Real Academia Española y el portal lexical del Centre National de Ressources Textuelles et Lexicales (CNTRL). 
etc.) - , con la finalidad de reducir las variables pragmáticas (Nord, 2003, p. 28).

Marianne Lederer divide los problemas léxicos como correspondencias y equivalencias: las primeras son las formas monorreferenciales que aparecen generalmente en los glosarios propios de cada disciplina, mientras que la noción de equivalencia surge de la aprehensión del sentido de la palabra en discurso (2003, pp. 18-20).

Ahora bien, en todo el proceso de traducción está presente el mecanismo de interferencia, que se hace visible en la fase de reformulación, especialmente con consecuencias sintáctico-discursivas que requieren poner en juego la subcompetencia bilingüe (Hurtado Albir, 2013, p. 395; Toury, 2004, pp. 345), como también la observancia de normas, según criterios de aceptabilidad o adecuación (Toury, 2004, pp. 97 y ss.).

Por último, al llegar a la fase del análisis justificativo y revisión, se considera relevante aplicar un criterio integrador que conjugue los niveles peritextuales y textuales (Larose, 1989, pp. 222-230).

Luego de estas reflexiones, cabe preguntarse qué caracteriza a la traducción de las ciencias sociales y humanas. Desde un nivel conceptual, estos textos se nutren de realidades locales, en oposición a la universalidad de las nociones intercambiadas por las ciencias naturales y formales, lo cual redunda en un mayor desafío en la búsqueda de equivalencias discursivas, en lugar de correspondencias monorreferenciales. A nivel de la expresión, además de la función referencial propia de los escritos especializados, estos textos movilizan "funciones particulares del lenguaje como la expresiva, la apelativa, la metalingüística y la estética" (Marín y Aguilar, 2009 , p. 102). ${ }^{8}$

8 Las autoras trabajan con tres conceptos que recorren la literatura traductológica: la equivalencia, en términos de Jean Delisle; el conocimiento del
Los objetivos de este artículo son, por un lado, contextualizar y documentar las experiencias sistematizadas de la etapa final de formación de traductores universitarios, en especial traducciones del área de las ciencias sociales y humanas, tomando como insumo la descripción de los informes en los que el alumnado interviniente registra los problemas de traducción encontrados y fundamenta el proceso traductivo con base en los principios teóricos incorporados a lo largo de sus estudios. Por otro lado, exponer el análisis que realiza la cátedra Traducción Científico-Técnica en Francés 2 sobre las anomalías de reformulación asociadas al mecanismo de interferencia presente en el vaivén entre el texto terminado de partida y la primera traducción, cuyas consecuencias pueden involucrar niveles superiores al microtexto.

Este artículo presenta la siguiente organización: en primer lugar, contextualiza la formación en traducción científico-técnica del último año del Traductorado Público Nacional en Lengua Francesa, que se dicta en la Facultad de Humanidades y Ciencias de la Educación (FaHCE) de la Universidad Nacional de La Plata (Argentina) (UNLP). A continuación, presenta las experiencias de traducción de artículos científicos de ciencias sociales. ${ }^{9}$ Finalmente, analiza ejemplos

mundo, según Sperber y Wilson, y la noción de fidelidad, según Steiner (Marín y Aguilar, 2009, pp. 104-105).

9 Con "artículos científicos" nos referimos al concepto bajtiniano de género discursivo, que se reconoce por su relación con determinadas prácticas sociales, por su formato y por el predominio de una secuencia textual sobre otras (Narvaja de Arnoux et al., 2002, p. 40). Las formas convencionales que caracterizan a los géneros discursivos pueden diferir de lengua a lengua y de cultura a cultura, razón por la cual revisten importancia para la traductología (Hurtado Albir, 2013, p. 484). Por su parte, Alexánder Sánchez Upegui considera al artículo científico como un informe escrito evaluado por pares, editado y publicado, que brinda reflexiones, avances parciales o resultados finales 
de anomalías de esas traducciones, en gran parte ligadas a la visibilización de interferencias, que se leen como huellas de otro texto, dificultan el ajuste entre la expresión y el contenido, e inciden más allá del microtexto donde aparecen (Larose, 1989, pp. 222-230). Son transferencias negativas que pueden originarse en el sistema lingüístico meta, en nuestro caso el español, en el discurso original expresado en francés, o pueden ser bidireccionales, como vaivén entre el discurso de partida y las reglas del sistema de la lengua meta (Cagnolati, 2010, pp. 319 y 324; 2015, p. 72).

\section{La traducción científico-técnica en el tramo final de formación}

Siguiendo la tradición universitaria y la convicción de que práctica y teoría se complementan para lograr un perfil de egresado autónomo y crítico, la FaHCE de la UNLP ha diseñado programas de formación en traducción en los pares de lenguas francés/español e inglés/español, con contenidos teórico-prácticos. ${ }^{10}$ Para el tema que nos compete, nos ocupamos especialmente del segundo nivel de Traducción Científico-Técnica en Francés (тстғ2) y de las Prácticas en Traducción (PT).

que se desprenden de las etapas de un proyecto específico de investigación (2012, pp. 106).

10 El plan de estudios del Traductorado Público Nacional en Lengua Francesa tiene un tronco común con el profesorado y las licenciaturas de orientación lingüística y literaria. En particular, presenta materias específicas de traducción, con dos niveles de traducción jurídico-económica, dos niveles de traducción literaria, dos niveles de traducción científico-técnica y un nivel de interpretación. Esta formación culmina con las Prácticas en Traducción, en las que cada estudiante elige una orientación (jurídica, literaria o científico-técnica) (FaHCE, unLP, 2016).

\subsection{Segundo nivel de traducción científico- técnica en francés}

Es un curso anual de 32 semanas, lo que representa 192 horas de clases, distribuidas en 128 horas teórico-prácticas y 64 horas prácticas. ${ }^{11}$

En тстF2, se refuerzan y amplían diversos tópicos teóricos desarrollados en cursos de traducción anteriores, en especial el primer nivel de traducción científico-técnica en francés (тСтF1), ${ }^{12}$ y se los trata en permanente diálogo con la práctica, o bien se parte de la práctica y se busca una teoría que la explicite (Lederer, 2003).

Estos tópicos se ocupan de la evolución de la traducción como proceso, producto y función, tomando enfoques cognitivos de la teoría interpretativa, el análisis de la traducción escrita (Delisle, 1984), los aportes sobre la interfaz comprensión/reformulación o modelo de la doble hélice (Dancette, 1995), la comprensión flotante (Gile, 1986), la interferencia en traducción (Toury, 2004) y los desarrollos acerca de las anomalías en la traducción de textos pragmáticos que hemos realizado (Cagnolati, 2010, 2015). También se revisan aspectos socioculturales, según las normas de Gideon Toury (2004); procedimentales, siguiendo a Daniel Gouadec (1989) y Christiane Nord (2003); terminológicos, diferenciando los criterios de la teoría general de la terminología (Arntz y Picht, 1989) y de la teoría comunicativa de la terminología (Cabré, 1999).

La práctica que se desarrolla en TCTF 2 está planteada a partir de la traducción de textos pragmáticos o vehiculares (Delisle, 1984, p. 22; 2005, p. 12), de 800 a 2000 palabras, con encargos

11 Las clases exclusivamente prácticas están a cargo de la Prof. Trad. Daniela Spoto Zabala.

12 La TCTF1 tiene una carga horaria análoga a TCTF2 y está a cargo de la Dra. Ana María Gentile y de la Prof. Trad. Daniela Spoto Zabala. 
de traducción de géneros discursivos técnicos (folletos explicativos, manual de instrucciones, prospecto de medicamento, etc.) y científicos (artículos científicos, ponencias destinadas a congresos), o adaptaciones con cambio de género discursivo (artículo de divulgación especializada a resumen, por ejemplo), traducciones inversas o directas - determinando claramente el polo de llegada, generalmente el español rioplatense en el caso de traducciones directas-, con elaboración de glosarios de cuatro columnas (entrada, traducción, fuente, observaciones).

Con el propósito de interrelacionar la teoría y la práctica, cada encargo plantea la aplicación de un tema teórico, como la formulación de ejemplos de correspondencias y equivalencias del discurso sinecdóquico traducido, en términos de Marianne Lederer (2003); identificación de las características del género discursivo de trabajo, e identificación de textos paralelos aplicables a todo el proceso traductivo.

Las temáticas que se abordan al inicio del curso corresponden a las ciencias agrarias, meteorología y energías renovables. La segunda mitad del curso está dedicada a traducir textos de ciencias sociales y humanas.

Se concluye con un trabajo final integrador, que comprende las siguientes secciones: traducción de un texto especializado, como se ha definido en la introducción de este artículo, casi siempre del género discursivo "artículo científico"; glosario terminológico, basado en fuentes documentales seguras; fichas terminológicas, solo si es necesario establecer puntualmente el alcance de un término, y redacción de un informe individual, en el que cada estudiante explicita los principios teóricos que sustentan su traducción, identifica los problemas de la traducción, describe la metodología utilizada para resolverlos y da su opinión sobre la experiencia.

Tanto en esta instancia como en las Prácticas en Traducción, los textos son proporcionados por docentes e investigadores del área del conocimiento tratado, que actúan como especialistas de referencia en un ambiente de trabajo interdisciplinario y de colaboración.

\subsection{Prácticas en Traducción}

Las PT replican las características del trabajo final integrador mencionadas en тстF 2 , pero con textos especializados más extensos.

Estas prácticas están concebidas como un ejercicio interdisciplinario preprofesional, que se realiza en alguna de las orientaciones de la carrera, es decir, traducción jurídico-económica, literaria o científico-técnica, a elección de cada estudiante.

Dentro de la vasta área que compete a la traducción científico-técnica, nos vinculamos periódicamente con centros de investigación, cátedras o departamentos universitarios interesados en leer en español bibliografía en francés, exclusivamente con fines académicos, en los campos de sus especialidades. Los géneros discursivos más solicitados son los artículos científicos, las comunicaciones de congresos y los capítulos de libro. En menor medida, se han realizado traducciones al francés de artículos científicos o comunicaciones para congresos.

El material propuesto para traducir debe cumplir ciertos requisitos: temática del texto, género discursivo, extensión -entre 10000 y 15000 palabras, aproximadamente - y plazos de seguimiento y entrega. En cuanto a los primeros requisitos, no se pondera el grado de dificultad ${ }^{13}$ de los textos de trabajo, sino la idea de que cada traducción es un caso particular, en el que se amalgaman aspectos generales de cualquier proceso traductivo, resumidos en el

13 " [...] conviene recordar que la noción de 'dificultad' es primeramente un concepto relativo y muy lejos de ser absoluto. ¿Difícil para quién y en qué condiciones? ¿Para el alumno o el profesor? ¿Para el alumno de qué año o con qué formación o experiencia previa?” (Bastin y Pomerleau, 2017, p. 12). 
contacto de dos lenguas y dos culturas, y otros aspectos distintivos, estos últimos vinculados con las características del género discursivo, el encargo de traducción, el tipo de traducción solicitada, el conocimiento del campo de la especialidad, las normas de traducción tomadas en cuenta y el plazo asignado para la presentación definitiva de cada trabajo, que es el que necesitan los estudiantes del Traductorado para ganar experiencia en una traducción cuasiprofesional.

Las características antes señaladas implican una coordinación que vincula a las partes integrantes del trabajo interdisciplinario - estudiante, docente coordinadora, especialista- y sostiene el seguimiento con devoluciones regulares, para lograr el desarrollo y la culminación de la tarea.

Dicho esto, el producto definitivo es el que permite ponderar retrospectivamente la competencia traductora adquirida, las fortalezas y debilidades de quien tradujo y, desde esa perspectiva, cobra fuerza la idea de que la lectura y la revisión de las sucesivas versiones que cada estudiante entrega durante el proceso de traducción develan las facilidades y dificultades de cada trabajo y el compromiso por dar respuesta a las exigencias del encargo de traducción. Esto nos lleva a privilegiar las devoluciones reflexivas, argumentadas, que contribuyen con el estudiante del último año del Traductorado de Lengua Francesa a afianzar la lectura crítica y la autonomía, y a desestimar las prescripciones sin justificación. Con esa actitud y en cursos del último año de formación poco numerosos, como los que se dedican al par francés/español, la clase se convierte en taller (Hurtado Albir, 1996, p. 55).

Las devoluciones siguen una lógica inscripta en el modelo integrador de la traducción de Robert Larose (1989), con lo cual la calidad de una traducción será el resultado del tratamiento de los niveles que este autor propone: por un lado, el nivel peritextual, que reúne el objetivo del enunciador, el tenor informativo, el componente material y el trasfondo sociocultural del público destinatario; por otro, el nivel textual, inspirado en Teun van Dijk (1983), que relaciona la superestructura esquemática y la macroestructura semántica con las formas microestructurales de la expresión y del contenido (grafémicas, morfológicas, lexicológicas, sintácticas). ${ }^{14}$

Además, la confrontación del texto original y su traducción es la herramienta que permite monitorear la conservación de la información, inferir el vaivén entre los niveles textuales y peritextuales, y examinar las soluciones, particularmente las anomalías que visibilizan la interferencia, según los niveles comprometidos.

Para el estudio de las anomalías, entendemos la traducción al español como producción de primera mano; focalizamos los fragmentos cuya lectura es poco fluida, y organizamos los binomios español/francés para su análisis.

\section{La experiencia en traducción de textos de ciencias sociales y humanas en el último año del traductorado}

En el campo de las ciencias sociales y humanas, ${ }^{15}$ se han realizado traducciones colaborativas en la cátedra TCTF 2 y en las PT con cátedras del profesorado en ciencias de la educación, equipos de investigación sobre estudios de género y docentes-investigadores de bioética.

En este artículo nos concentramos en la traducción del francés al español de artículos científicos propuestos por el Laboratorio de

14 El criterio histórico de evaluación de la cátedra sigue esa lógica.

15 Además de traducciones de textos de ciencias sociales y humanas, se han realizado trabajos interdisciplinarios en otros campos, como matemática, ciencias agrarias y forestales, música. 
Estudios en Sociología y Economía del Trabajo (LESET) ${ }^{16}$ y seleccionados en la cátedra TCTF2, en función de su extensión y de los intereses personales de las alumnas. ${ }^{17}$ Los textos fueron:

- Texto 1: La perception des liens travail/ santé. Le rôle des normes de genre et de profession, de Paul Bouffartigue, Jean-René Pendariès y Jacques Bouteiller (2010). Traducción: La percepción de los vínculos trabajo / salud. El papel de las normas de género y de profesión.

- Texto 2: Le stress au travail, entre psycholositation et critique des conditions travail, de Paul Bouffartigue (2010). Traducción: El estrés en el trabajo, entre la psicologización y la crítica de las condiciones laborales.

- Texto 3: Jongleuses en blouse blanche : la mobilisation des compétences temporelles chez les infirmières hospitalières, de Paul Bouffartigue y Jacques Bouteiller (2003). Traducción: Malabaristas de guardapolvo blanco: la movilización de las competencias temporales en las enfermeras hospitalarias.

- Texto 4: "L'encampement " des réfugiés syriens au Moyen Orient : déborder les frontières de la vulnérabilité par le travail, de Mustapha El Miri y Delphine Mercier (2018). Traducción: "El encampamiento" de los refugiados sirios en Medio

16 El LESET funciona en la FaHCE, y forma parte del Instituto de Investigaciones en Humanidades y Ciencias Sociales, de doble dependencia: ConICET-UNLP. La especialista de referencia es la Dra. Mariana Busso, con quien coordinamos los textos para traducir, su extensión, los plazos y el seguimiento del alumnado de TCTF2.

17 Las alumnas que participaron en estos trabajos son Carolina Aguilar, Natasha Kusmusk, Marcela Supera y Lucía Santa María.
Oriente: superar las fronteras de la vulnerabilidad a través del trabajo. ${ }^{18}$

Las traducciones realizadas cumplen con los requisitos que asigna la cátedra: son integrales; transmiten datos, informaciones, contenidos y formas del documento original, respetando las características y las normas del documento fuente, pero atendiendo a las exigencias que se relacionan con eventuales adaptaciones culturales del documento final, con la lógica propia del campo del saber dentro del cual se inscribe el texto fuente, y la calidad lingüística y la coherencia terminológica convenidas en el proyecto de traducción (Gouadec, 1989, p. 28).

\subsection{Los informes de traducción}

Los informes de traducción elaborados por las alumnas graduadas el mismo año de realización del trabajo final de TстF 2 o de las PT, o por graduarse, constan de tres partes, como se explica a continuación.

\subsubsection{Primera parte}

Esta es introductoria y contextualiza la actividad, Pт o trabajo final integrador de тстF2. Por un lado, informa sobre los objetivos de la actividad, es decir, traducir un artículo científico que asegure la coherencia y la cohesión, y permita una lectura fluida en el polo meta (rioplatense), contribuyendo con la recepción en el ámbito académico de la FaHCE; por otro, presenta las características textuales y paratextuales de cada artículo de trabajo.

En cuanto a las temáticas, el texto 1 aborda las representaciones de la relación entre trabajo y salud en los trabajadores tercerizados y en las

18 Los textos originales propuestos por el LESET para su traducción provienen de grupos de investigación del Laboratoire d'économie et de sociologie du travail (LEST), dependiente del Centre national de la recherche scientifique (CNRS). Véase LEST (s. f.). 
trabajadoras de la salud; el texto 2 trata sobre la situación de estrés en las trabajadoras de la salud; el texto 3 estudia la complejidad del régimen horario de las enfermeras hospitalarias, y el texto 4 analiza el tráfico clandestino de migrantes y la explotación de los trabajadores en sectores económicos del país de acogida.

\subsubsection{Segunda parte}

Esta sección está dedicada a presentar los enfoques traductológicos o terminológicos que sustentan cada trabajo, para lo cual las alumnas aplican los contenidos que incorporaron durante la carrera. En general, se basan en los contenidos teóricos del análisis del proceso de traducción y los niveles de manejo de la lengua de Delisle (1984, pp. 69-125); los criterios tourianos de aceptabilidad y adecuación; la aplicación del concepto de texto paralelo de Nord (2003, pp. 23-39); la problemática de la interferencia, que se desarrolla en las clases de тстғ2; los posicionamientos terminológicos clásicos o sociolingüísticos, estos últimos especialmente discutidos en тстF1, y las técnicas de traducción, según la clasificación de Hurtado Albir (2013, pp. 268-272).

Mediante el establecimiento de relaciones con los conceptos teóricos seleccionados, las alumnas identifican y caracterizan las actividades que realizaron en cada etapa del proceso de traducción:

- La primera etapa está dedicada a la lectura, la detección de problemas de comprensión y las estrategias para solucionarlos.

La lectura del texto fuente es pormenorizada, con un intervalo de decantación antes de comenzar a traducir.

La detección de problemas en los textos de ciencias sociales está vinculada con el conocimiento del tema, por lo que implica el manejo de los conceptos de la especialidad y la terminología que los representa; pero, sobre todo, con el uso de léxico cotidiano, que puede acarrear ambigüedad al intentar reformularlo.

Para su solución, además de la consulta en diccionarios, la cátedra privilegia la búsqueda en textos paralelos (en términos de Nord, 2003, p. 28), con especial atención en que sean análogos los factores de las situaciones comunicativas de uso del texto de partida y de los textos buscados, ya sea para documentarse o resolver problemas léxicos. Por ejemplo, en el campo de la sociología del trabajo, artículos científicos producidos en el LEST-CNRS (Francia) y artículos científicos producidos por el LESET-CONICET (Argentina).

Un momento especial en esta primera etapa fue la entrevista con la especialista de referencia, fundamental para asistir a las estudiantes en los casos problemáticos e indicar fuentes confiables de consulta que les permitiera elaborar un glosario abierto y enriquecido en posteriores traducciones.

- La segunda etapa corresponde a la reformulación. Además de resolver problemas de correspondencias y equivalencias, las alumnas destacan la minuciosa lectura de largas oraciones, no siempre articuladas con la puntación o los medios cohesivos esperados. Señalan también un uso reiterado de formas enfáticas (C'est... que/qui), de gerundios, como también de regímenes verbales (permettre de), cuya resolución requiere que se apliquen conocimientos contrastivos de los sistemas lingüísticos en contacto.

- La tercera etapa está dedicada a la relectura de control, con la finalidad de detectar errores de traducción o de distracción, antes de enviar el trabajo a la docente coordinadora para la primera lectura externa. Durante esta etapa, se llevan a cabo varias relecturas e intercambios entre alumnas y docente, antes de 
enviar la traducción a la especialista. Siguen luego relecturas y discusiones entre alumnas, docente coordinadora y especialista, hasta la aceptación final.

\subsubsection{Tercera parte}

En esta sección del informe de traducción se puntualizan los problemas encontrados, lingüísticos, discursivos y culturales, con ejemplificación y estrategias para su resolución.

- En el plano terminológico, las alumnas discuten casos que no pudieron ser resueltos mediante la consulta a las fuentes clásicas.

El adjetivo professionnel(lle) (Ejemplo 1). En Francia, el sustantivo profession remite al mundo del trabajo en general; en Argentina, el ejercicio de una profesión implica acreditar una formación de nivel terciario. En cuanto al adjetivo profesional, su uso es más amplio que el del sustantivo y coexiste con el adjetivo laboral. En textos paralelos, aparecen varias combinaciones: riesgos laborales, enfermedades profesionales, trayectoria profesional, vida laboral, actividad laboral, etc. (Neffa, 2015).

Para la resolución de estos casos, es decisiva la opinión de la especialista de referencia.

\section{Ejemplo 1}

\begin{tabular}{|c|c|}
\hline Francés & Español \\
\hline $\begin{array}{l}\text { risques professionnels (Texto } 1 \text {, } \\
\text { p. 249). }\end{array}$ & riesgos laborales \\
\hline $\begin{array}{l}\text { vie professionnelle (Texto 1, } \\
\text { p. 250). }\end{array}$ & vida laboral \\
\hline
\end{tabular}

El sustantivo soignantes (Ejemplo 2). En el texto fuente, este término designa el conjunto de enfermeras y auxiliares de un hospital. En la realidad argentina local, el sustantivo cuidadoras no cubre a ese colectivo. Se optó, entonces, por consultar una fuente directa, un hospital de la ciudad de La Plata, pero no se obtuvo

\section{Ejemplo 2}

\begin{tabular}{|c|c|}
\hline Francés & Español \\
\hline $\begin{array}{l}\text { Les soignantes (Texto 1, } \\
\text { p. 260) }\end{array}$ & $\begin{array}{l}\text { Las trabajadoras de la } \\
\text { salud }\end{array}$ \\
\hline
\end{tabular}

Ejemplo 3

\begin{tabular}{|c|c}
\multicolumn{1}{c}{ Francés } & \multicolumn{1}{c}{ Español } \\
$\begin{array}{l}\text { catégories socioprofessionnelles } \\
\text { (Texto 1, p. 260) }\end{array}$ & $\begin{array}{l}\text { categorías } \\
\text { ocupacionales }\end{array}$
\end{tabular}

Ejemplo 4

\begin{tabular}{|c|c|}
\hline Francés & Español \\
\hline $\begin{array}{l}\text { Tel ce conducteur routier } \\
\text { qui, sous la pression } \\
\text { explicite de son épouse } \\
\text { - il est vrai nettement } \\
\text { plus diplômée et mieux } \\
\text { rémunérée que lui - a du } \\
\text { renoncer à son métier } \\
\text { pour se sédentariser } \\
\text { (Texto 3, p. 7). }\end{array}$ & $\begin{array}{l}\text { Es el caso de un chofer } \\
\text { de camión que - } \\
\text { bajo la presión de su } \\
\text { cónyuge que tenía } \\
\text { mayor formación y } \\
\text { remuneración que él- } \\
\text { tuvo que renunciar a } \\
\text { su actividad y se volvió } \\
\text { inactivo. }\end{array}$ \\
\hline
\end{tabular}

una designación que englobara a enfermeras y auxiliares. La especialista de referencia derivó la consulta a quienes estudian temas laborales vinculados con la salud y la propuesta obtenida fue trabajadoras de la salud. ${ }^{19}$

El sintagma nominal catégories socioprofessionnelles (Ejemplo 3). Las nomenclaturas pueden compararse consultando las páginas de dos institutos equivalentes: en Francia, el Institut National de la Statistique et des Études Économiques (2017), y en Argentina, el Instituto Nacional de Estadística y Censos (2011).

E1 sustantivo épouse (Ejemplo 4). El texto francés utiliza épouse, cuya correspondencia en español es esposa. En cambio, los textos locales de ciencias sociales y humanas prefieren la palabra invariable cónyuge, tanto para el femenino (la cónyuge) como para el masculino (el cónyuge).

19 Sofía Malleville es becaria doctoral con lugar de trabajo en el LESET. Con posterioridad a la entrega de la traducción, se registra la designación trabajadoras de la salud en East, Laurence y López (s. f.). 
- En el plano del léxico general, las alumnas identifican numerosas palabras de uso corriente cuya designación varía según el contexto. Para ilustrarlo, seleccionamos dos, la primera porque su uso es frecuente en los textos generales y especializados; la segunda, porque moviliza la búsqueda en el registro familiar, fenómeno natural en estos textos que se nutren de las subjetividades de los grupos sociales estudiados y son casi inexistentes en los textos de las disciplinas naturales y formales.

El adjetivo faible (Ejemplo 5). Este se utiliza en contextos diversos. Es fácilmente comprensible, pero su reformulación es algo laboriosa, puesto que la diversidad de coocurrentes en francés no se corresponden necesariamente con los del adjetivo débil en español. En el Ejemplo 5, la coocurrencia con la palabra forma es natural. En cambio, cuando faible modifica la palabra différence, la reformulación en español apela a otros adjetivos según el contexto: poca, mínima, etc., como se muestra en el Ejemplo 6.

\section{Ejemplo 5}

\begin{tabular}{|c|c|}
\hline Francés & Español \\
\hline $\begin{array}{l}\text { [...] la forme « faible » de } \\
\text { la non-reconnaissance } \\
\text { de liens entre travail- } \\
\text { santé, ou « forte » de } \\
\text { la revendication d'une } \\
\text { influence positive } \\
\text { (Texto 1, p. } 253 \text {. }\end{array}$ & $\begin{array}{l}\text { [...] la forma "débil" } \\
\text { del no reconocimiento } \\
\text { de vínculos entre } \\
\text { trabajo y salud, o a la } \\
\text { forma "fuerte" de la } \\
\text { reivindicación de una } \\
\text { influencia positiva. }\end{array}$ \\
\hline
\end{tabular}

\section{Ejemplo 6}

\begin{tabular}{l:l}
\multicolumn{1}{c}{ Francés } & \multicolumn{1}{c}{ Español } \\
$\begin{array}{l}\text { Faible parmi les ouvriers, } \\
\text { la différence entre les } \\
\text { sexes est très marquée } \\
\text { dans les trois autres } \\
\text { grandes catégories }\end{array}$ & Si bien entre los obreros \\
(Texto l, p. 254). & la diferencia entre los \\
notable en las otras tres & grandes categorías.
\end{tabular}

El sustantivo débrouille y el verbo se débrouiller. Ambas expresiones, familiares al texto fuente, se reformularon con elementos de la cultura receptora de un nivel lingüístico análogo: rebusque; arreglárselas.

Es necesario anotar que la consecuencia del fenómeno sinecdóquico de una traducción se muestra en los textos traducidos de ciencias sociales y humanas, especialmente en el terreno de las equivalencias, como se ha ejemplificado anteriormente. La explicación que las acompaña excede la información que aportan los diccionarios y se focaliza en estrategias de análisis discursivo en relación con el contexto textual y temático, con el rastreo en textos paralelos (ejemplos 3, 4) y, en ocasiones, con el asesoramiento de la especialista, para llegar a la reformulación de conceptos con las designaciones pragmáticamente esperadas (ejemplos 1, 2).

En cuanto al léxico general, los ejemplos 5 y 6 se focalizan en la polisemia, cuyo tratamiento necesita un análisis del contexto general de la temática tratada, pero además microtextual; el Ejemplo 7 sustenta la idea de la amplitud del lenguaje utilizado en los textos del área estudiada, reveladores de funciones que van más allá de la referencial. En este caso, la activación de la subcompetencia bilingüe parte de la identificación del registro familiar, la función expresiva de la débrouille y la habilidad de la traductora para restablecer esos parámetros en la equivalencia rebusque.

\section{Ejemplo 7}

\begin{tabular}{l:l}
\multicolumn{1}{c}{ Francés } & \multicolumn{1}{c}{ Español } \\
\hline $\begin{array}{l}\text { S'insérer dans les } \\
\text { interstices de l'économie }\end{array}$ & $\begin{array}{l}\text { Insertarse en los } \\
\text { intersticios de la } \\
\text { de la débrouille (Texto 4, } \\
\text { p. 11). }\end{array}$ \\
\hdashline $\begin{array}{l}\text { Dans les faits les réfugiés } \\
\text { se débrouillent par eux- }\end{array}$ & $\begin{array}{l}\text { De hecho, los refugiados } \\
\text { mêmes en s'insérant }\end{array}$ \\
dans les interstices des & mismos insertándose \\
économies formelles & en los intersticios de las \\
et informelles très & economías formales \\
développées dans les & informales, muy \\
pays d'accueil (Texto 4, & desarrolladas en los \\
p. 8). &
\end{tabular}




\subsection{Balance de la experiencia}

En este apartado, las intervinientes ponderan su experiencia y la expresan mediante opiniones generales sobre el proyecto y sobre aspectos idiosincrásicos de los textos traducidos.

\subsubsection{Opiniones generales sobre el proyecto}

Los proyectos de traducción especializada son un espacio ideal para un trabajo colaborativo de equipo, con devoluciones finales de lectoras expertas. Además, estos trabajos dan la posibilidad de reactivar conceptos traductológicos adquiridos, relacionarlos con la práctica e indagar sobre la información que un traductor deber tener para publicar en una revista especializada, comenzando por la autorización de los autores y las normas de publicación de la misma.

Dentro del proceso traductivo, las estudiantes puntualizan los tópicos siguientes: las sucesivas lecturas analíticas del texto fuente, que demuestran la importancia de cada una de las palabras en la construcción de sentido; las lecturas complementarias de textos paralelos en español rioplatense; la búsqueda terminológica en fuentes seguras, que queda registrada en un glosario colaborativo; un particular esfuerzo en la reformulación, para lograr fluidez sin correr el riesgo de perder el sentido del texto; la traducción casi literal de algunos fragmentos, con miras a respetar el mensaje de los autores, aun a riesgo de que la producción se aleje del polo meta.

\subsubsection{Identificación de los aspectos idiosincrásicos del corpus textual}

A continuación agrupamos los aspectos más destacados de los informes de las intervinientes de la experiencia:

1. Los textos de ciencias sociales leídos, tanto el traducido como los textos paralelos que amplían la competencia extralingüística, relacionan disciplinas diversas y se nutren de sus terminologías (psicología, historia, medicina, etc.).

2. Los textos de ciencias sociales recurren con frecuencia al uso de palabras comunes, cuya traducción puede acarrear problemas de inestabilidad, ambigüedad o de desajustes pragmáticos.

3. Cada texto científico tiene su propia forma y características, y la persona que traduce debe tenerlas en cuenta, y preservar ciertos estereotipos propios del texto original, como las observaciones de campo, las entrevistas cualitativas, los datos estadísticos, los cuadros de referencia, etc.

4. Se identifican párrafos extensos, sintaxis compleja con oraciones sin puntuación o marcas cohesivas, lo que exige una atenta reformulación orientada al lector local, sin descuidar la información aportada por el texto fuente, en busca de un equilibrio entre el polo de partida y el de llegada.

En cuanto al balance general, las alumnas valoran positivamente la experiencia interdisciplinaria con las diferentes áreas académicas de la FaHCE como práctica para la formación en traducción, lo que "permite salir de nuestro compartimento, produciendo un intercambio de saberes, que van más allá del aprendizaje individual", según las palabras de una de las últimas egresadas. ${ }^{20}$

Acerca de los aspectos idiosincrásicos enumerados, el primer punto marca la matriz interdisciplinaria de las ciencias sociales y humanas, que se manifiesta en estos textos, asociada al uso de correspondencias portadoras de conceptos monorreferenciales de diversos

20 Lucía Santa María se graduó en 2020. 
campos del conocimiento, entre otros, la medicina (pathologies ostéoarticulaires/patologías osteoarticulares, texto 2 ; silicose/silicosis, texto 1), la historia (l'hygiénisme industriel/higienismo industrial, texto 1) y la psicología (stress/ estrés, texto 2). Naturalmente, son frecuentes las correspondencias de la sociología y la economía del trabajo (ergonomique/ergonómico, organisation patronale/organización patronal, salarié/asalariado, texto 2 ; taylorisation/taylorización, texto 3 ).

El segundo punto, relacionado con el léxico, queda subsumido en el tercero, este último centrado en la intertextualidad, que se pone en evidencia en la cita de encuestas, entrevistas, opiniones (Je suis un bosseur/Soy un laburador; Les gens veulent du beurre et l'argent du beurre... Les horaires, ça fait partie du métier !/La gente exige demasiado, todo no se puede... ¡Los horarios son parte del trabajo! Si no, que hagan otra cosa, texto 1). En el último ejemplo, la idea se comprende, pero la función expresiva del refrán se ha visto menguada y la traducción necesitaría restituirla pragmáticamente, por medio de expresiones familiares de la lengua-cultura de llegada, cuyo semantismo se remonta a datos sociohistóricos y regionales, como "la gente quiere el oro y el moro" o "la gente quiere la chancha y los veinte", ${ }^{21}$ este último de origen rioplatense.

Las entrevistas y encuestas citadas en estos textos tratan sobre temáticas laborales con opiniones directas de las personas informantes, que luego analizan los autores en una relación metatextual, mediante conceptos compartidos o discutidos por la comunidad especializada. Si bien la función principal de los textos especializados es referencial, estos se ven permeados por otras funciones, en la voz de los investigadores.

21 La expresión completa es "la chancha y los veinte y la máquina de hacer chorizos".
Por ejemplo, el texto 3 trata sobre la complejidad de la administración del tiempo de las enfermeras hospitalarias, y en los últimos tres renglones del artículo, los autores adhieren al avance de su profesionalización. Para expresarlo, contraponen el futuro de la enfermería con un modelo ya anticuado l'ancien modèle de la "bonne " et de la "nonne", en el que, además del semantismo de cada palabra, los autores recurren al poder evocador y rimado de bonne, nonne. En el proceso de reformulación de ese enunciado se han identificado las funciones expresiva y estética, lo que ha dado como producto el viejo modelo de "la hacendosa religiosa", mediante la transposición del sustantivo bonne por el adjetivo hacendosa y el equivalente de nonne/religiosa, que logra el efecto de rima.

\section{Identificación y análisis de anomalías en la producción discursiva de traducción}

Entendemos por anomalía todo elemento que incide negativamente en la lectura de una traducción, e ilustramos ese concepto con dos ejemplos, seleccionados según los criterios de evaluación históricos de la cátedra, que encuentran la mayor cantidad de anomalías en el nivel morfosintáctico (Alliaud et al., 2001, pp. 96-115; Spoto Zabala, 2020, pp. 110-112).

Transcribimos los ejemplos como binomios constituidos por los textos traducidos (тT) y los textos originales (то). A la izquierda, se consigna la traducción, y a la derecha, el texto de partida, con la finalidad de representar espacialmente la primera lectura realizada por el público receptor, que accede al conocimiento a través de la versión en español.

En la columna izquierda, se transcribe la primera reformulación con la anomalía y más abajo la producción final que la resuelve.

Sigue luego el análisis, que parte del reconocimiento de la anomalía, su posible causa y el 


\section{Ejemplo 8}

\begin{tabular}{|c|c|}
\hline Tा español & To francés \\
\hline $\begin{array}{l}\text { Primera reformulación } \\
\text { Según M. Loriol (2005), las trabajadoras de la salud } \\
\text { son un buen ejemplo de la psicologización de } \\
\text { las dificultades laborales. Desde su formación, la } \\
\text { psicología es la disciplina que sostiene teóricamente } \\
\text { la visión de su capacidad laboral, la de un "papel } \\
\text { propio" que apunta a un "enfoque global" del } \\
\text { enfermo (el resaltado es mío). }\end{array}$ & \multirow[t]{2}{*}{$\begin{array}{l}\text { Selon M. Loriol (2005), les soignantes sont un bon } \\
\text { exemple de la psychologisation des difficultés } \\
\text { professionnelles. La psychologie est la discipline qui } \\
\text { soutient théoriquement, dès leur formation, leur vision } \\
\text { de leur compétence professionnelle, celle d'un « rôle } \\
\text { propre » visant « l'approche globale » du malade } \\
\text { (Texto 2, p. 6). }\end{array}$} \\
\hline $\begin{array}{l}\text { Producción final } \\
\text { Según M. Loriol (2005), las trabajadoras de la salud } \\
\text { son un buen ejemplo de la psicologización de } \\
\text { las dificultades laborales. Desde su formación, la } \\
\text { psicología es la disciplina que sostiene teóricamente } \\
\text { la visión de estas trabajadoras sobre su capacidad } \\
\text { laboral, la de un "papel propio" que apunta a un } \\
\text { "enfoque global" del enfermo. }\end{array}$ & \\
\hline
\end{tabular}

cálculo de las consecuencias de la información proporcionada.

Este análisis está organizado en tres partes: 1) comparación del binomio TT-TO, en donde se muestran las anomalías microtextuales que inciden en desajustes entre la expresión y el contenido; 2) resultado de la primera reformulación, en donde se consigna si hubo visibilidad de interferencia, el origen de la interferencia y la incidencia peritextual, y 3) producción final, en la que se determina si mejoró la primera reformulación. ${ }^{22}$

\subsection{Ejemplo 8}

\subsubsection{Comparación del binomio тT-то}

La segunda oración del то comienza con el sujeto La psychologie e intercala el adjunto prepositivo dès leur formation, repitiendo inmediatamente dos veces el determinante posesivo leur. E1 Tт encabeza la segunda oración con el adjunto prepositivo Desde su formación, con el determinante posesivo $s u$, que remite a un referente transfrástico. Sigue luego el sujeto la psicología y más adelante el posesivo incluido en el sin-

22 Es una adaptación de nuestras guías de análisis previas (Cagnolati, 2010, pp. 146-149, 2015, pp. 62-65). tagma preposicional de su capacidad laboral. Una lectura superficial y mecánica podría confundir el referente de este posesivo de tercera persona.

Contrastivamente, el francés ofrece un paradigma de posesivos más rico que el español en las posibilidades de la tercera persona (son, sa, ses, leur, leurs) y de la segunda persona (votre, vos). Esta característica no pertenece al español local de Argentina, que dispone morfológicamente para expresarlas solo del posesivo singular su y el plural sus.

\subsubsection{Resultado de la primera versión}

- Visibilidad de interferencia bidireccional: a partir del то se restituyen automáticamente las reglas del sistema lingüístico del español, materializadas en este caso en la reiteración del posesivo su.

- Producción palimpséstica con incidencia peritextual en el trasfondo sociocultural del público destinatario.

\subsubsection{Producción final}

Con el objetivo de facilitar la lectura y dar mayor cohesión al fragmento, se retomó el referente transfrástico estas trabajadoras. 


\section{Ejemplo 9}

\begin{tabular}{|c|c|}
\hline пा español & To francés \\
\hline $\begin{array}{l}\text { Primera reformulación } \\
\text { Por ello, es posible que el mismo tipo de limitaciones } \\
\text { laborales esté mucho más asociado de manera } \\
\text { subjetiva a las responsabilidades de las mujeres que a } \\
\text { las de los hombres. Por ejemplo, los horarios imprevistos } \\
\text { o irregulares las afectan con más frecuencia (Bué y } \\
\text { Coutrot, 2009) y las consecuencias de ello son tan } \\
\text { negativas para las mujeres que terminan asumiendo } \\
\text { en gran parte el trabajo doméstico. Sin embargo, se } \\
\text { percibe la dificultad que tienen en "conciliar" vida } \\
\text { familiar y vida laboral, e incluso terminan abocándose } \\
\text { más a las responsabilidades de la familia que al trabajo } \\
\text { (el resaltado es mío). }\end{array}$ & \multirow[t]{2}{*}{$\begin{array}{l}\text { Ainsi, le même type de contraintes professionnelles est } \\
\text { probablement davantage associé subjectivement aux } \\
\text { responsabilités domestiques chez les femmes que chez } \\
\text { les hommes. Par exemple, les horaires imprévisibles ou } \\
\text { irréguliers les concernent plus souvent (Bué et Coutrot, } \\
\text { 2009), leurs implications seraient d'autant plus négatives } \\
\text { pour elles qu'elles assument l'essentiel du travail } \\
\text { domestique, mais elles seront probablement souvent } \\
\text { perçues au travers du thème de la difficulté de « } \\
\text { concilier » vie familiale et vie professionnelle, voire plutôt } \\
\text { imputées aux responsabilités familiales elles-mêmes } \\
\text { qu'au travail en tant que tel (Texto 1, p. 257). }\end{array}$} \\
\hline $\begin{array}{l}\text { Producción final } \\
\text { Por ejemplo, los horarios imprevistos o irregulares las } \\
\text { afectan con más frecuencia (Bué y Coutrot, 2009) y } \\
\text { las consecuencias son muy negativas para las mujeres } \\
\text { porque son ellas las que terminan asumiendo en gran } \\
\text { parte el trabajo doméstico. Lo curioso es que a ellas } \\
\text { se las suele percibir desde la dificultad que tienen en } \\
\text { "conciliar" vida familiar y vida laboral, e incluso desde } \\
\text { la obligación de abocarse a las responsabilidades de } \\
\text { la familia y no del trabajo. }\end{array}$ & \\
\hline
\end{tabular}

\subsection{Ejemplo 9}

\subsubsection{Comparación del binomio tT-тo}

Se observa una larga oración del то Par exemple, les horaires... que fue dividida en el тт para facilitar la lectura Por ejemplo, los horarios... Sin embargo, se percibe...

En la oración que comienza con Por ejemplo, los horarios imprevistos... del TT, se advierte la anomalía sintáctica en la reformulación del comparativo d'autant plus négatives pour elles qu'elles... que no explicita la conjunción causal.

La oración del тт iniciada con Sin embargo, se percibe... presenta una reformulación que se aparta de la sintaxis del to y no logra completar el sentido que este ofrece. Una de esas anomalías es la elipsis del sujeto en la proposición relativa que tienen en "conciliar" vida familiary vida laboral y en el enunciado e incluso terminan abocándose.

\subsubsection{Resultado de la primera reformulación}

- Visibilidad de interferencia de la lengua materna: la producción sigue automáticamente las reglas del sistema lingüístico del español, que tienden a la elipsis del sujeto.

- Resolución gramatical sin activación de conocimientos constrastivos.

- Producción palimpséstica con descenso cohesivo e incidencia peritextual en la conservación del tenor informativo.

\subsubsection{Producción final}

Se construye el comparativo con explicitación de la causa porque.

Se explicita el agente mujeres, ellas. Se profundiza la comprensión de los matices que subyacen en el to y la producción discursiva los expresa. 
Es de notar que las anomalías encontradas en el nivel microtextual se leen como huellas de un texto ajeno, que obstaculizan la fluidez del texto traducido y generan una disminución de su libertad, con la consiguiente necesaria consulta del To, que es lo que denominamos "producción palimpséstica" (Cagnolati, 2010, p. 166). Generalmente, son transferencias negativas, que se originan en el sistema lingüístico meta, o pueden ser bidireccionales, cuando el discurso de origen ha sido excesivamente respetado, con una producción basada en una correspondencia solo lingüística, vinculada con un modo de procesamiento textual ascendente. La identificación de estos problemas y la reflexión sobre sus consecuencias en niveles peritextuales contribuyen a controlar la producción discursiva.

\section{Conclusiones}

En este artículo hemos expuesto la localización de la traducción de las ciencias sociales y humanas en el último tramo de formación de traductores universitarios en idioma francés en la FaHCE de la unLP, y también hemos documentado las experiencias interdisciplinarias y sistematizadas que se han desarrollado en los últimos años entre la cátedra y el LESET.

Los informes de traducción analizados identifican las características del género discursivo del corpus textual: artículos científicos del área de las ciencias sociales, con secciones propias de las investigaciones sociológicas encuestas, entrevistas cualitativas, datos estadísticos, cuadros de referencia-, secuencias dominantes argumentativas que sostienen la investigación, combinadas con secuencias descriptivas y explicativas de las situaciones sociales estudiadas en cada artículo.

Asimismo, se ha observado la intertextualidad en las secciones que citan encuestas y entrevistas sobre temáticas laborales, con opiniones de las personas informantes. Esas secciones presentan expresiones familiares o refranes cuya función expresiva debe ser trabajada especialmente, para ser restituida en la lengua-cultura de llegada. En cuanto a las opiniones directas, estas son analizadas por los autores de cada artículo en una relación metatextual, mediante conceptos compartidos o discutidos por la comunidad especializada. Si bien la función principal de los textos especializados es referencial, el corpus original también consigna funciones expresivas y estéticas en la voz de los investigadores.

El corpus textual trabajado muestra la matriz interdisciplinaria de las ciencias sociales y humanas, que se manifiesta asociada al uso de correspondencias portadoras de conceptos monorreferenciales de diversos campos del conocimiento, entre otros, medicina, historia, psicología. Naturalmente, también son frecuentes las correspondencias del área de la sociología y la economía del trabajo, todas ellas de resolución relativamente sencilla, por medio de la búsqueda en diversas fuentes (diccionarios, bases de datos terminológicos, textos paralelos).

De todos modos, los informes puntualizan problemas de equivalencias en el corpus trabajado, cuya solución se focaliza en estrategias de análisis discursivo en relación con el contexto textual y temático, en el rastreo en textos paralelos (ejemplos 3, 4) y, en ocasiones, es determinante el asesoramiento de la especialista (ejemplos 1, 2). Además, se comentan problemas de polisemia (ejemplos 5 y 6) que fueron tratados con un análisis del contexto general de la temática tratada y del nivel microtextual.

Este artículo expone, además, el análisis que realiza la cátedra sobre las anomalías de reformulación asociadas al mecanismo de interferencia. Lo hemos ilustrado con el análisis de dos ejemplos de anomalías sintáctico-discursivas, puesto que, cuantitativamente, son las frecuentes, según las evaluaciones históricas llevadas a cabo por la cátedra. 
En particular, revisamos la visibilización de interferencias, manifiestas en la lectura como huellas de otro texto que dificultan el ajuste entre la expresión y el contenido, como surge en las primeras reformulaciones de los ejemplos compartidos, e inciden en los niveles peritextuales de transmisión de información y de aceptación en el público receptor.

Teniendo en cuenta las especificidades señaladas en el corpus textual traducido, el proceso de la traducción de textos de ciencias sociales y humanas está inscripto en la problemática traductológica general, que supone una aproximación lingüístico-contrastiva de dos lenguas-cultura en contacto, sobrepasada por la dinámica propia del discurso que el traductor va construyendo en la lengua y la cultura de llegada.

La combinación entre especialidad y cotidianeidad requiere un proceso traductivo que armonice la creatividad con el rigor científico, la búsqueda en fuentes seguras que reflejen la evolución y el uso concreto de los conceptos, y la identificación de recursos lingüísticos que expresen funciones más allá de la referencial y activen una actitud lúdica y controlada a la vez, a través de la cual quien traduce logre una producción discursiva equifuncional.

\section{Referencias}

Arntz, R. y Picht, H. (2010 [2008]). Introducción a la terminología (A. de Irazazábal et al., Trads.). Pirámide.

Bastin, G. y Pomerleau, M. (2017). La traducción especializada en la historia de la traducción y de la revista Meta. Sendebar, (N. ${ }^{\circ}$ Ext. 28), 9-30. https:// dialnet.unirioja.es/revista/2002/A/2017

Berrichi, A. (2012). La traduction en sciences sociales. Traduire, (227), 16-28. https://doi. org/10.4000/traduire. 467

Bouffartigue, P. (2010). Le stress au travail, entre psycholositation et critique des conditions travail [Séance de congrès]. Xvirème congrès de l'AIs, juillet 2010, Suède. https://halshs.archives-ouvertes.fr/halshs-00442122/document
Bouffartigue, P., Pendariès, J.-R. y Bouteiller, J. (2010). La perception des liens travail/santé. Le rôle des normes de genre et de profession. Revue Française de Sociologie, 51, 247-280. https://doi.org/10.3917/rfs.512.0247

Bouffartigue, P. y Bouteiller, J. (2003). Jongleuses en blouse blanche : la mobilisation des compétences temporelles chez les infirmières hospitalières. Actes des Ixèmes Journées de Sociologie du travail "Contraintes, normes et compétences au travail : les régimes de mobilisation", Atelier 1 «Evaluation des situations de travail », Centre Pierre Naville, Travail et mobilités, Paris, 2728 novembre 2003, Paris, France. https:// halshs.archives-ouvertes.fr/halshs-00007507/ document

Cabré, M. T. (1999). La terminología. Antártida/ Empúries.

Cagnolati, B. E. (2010). La producción discursiva en la traducción de textos pragmáticos francés/ castellano: estudio traductológico a través de los enunciados elípticos con anomalías [Tesis doctoral, Universidad Nacional de la Plata]. http://www.memoria.fahce.unlp.edu.ar/ tesis/te.359/te.359.pdf

Cagnolati, B. E. (2015). Interferencia en la traducción francés/español de textos de ciencias sociales. Hikma, 14, 55-74. https:// doi.org/10.21071/hikma.v14i.5200

Centre National de Ressources Textuelles et Lexicales (CNTRL). [Página web]. https://www.cntrl.fr/

Consejo Nacional de Investigaciones Científicas y Técnicas (CONICET). (s. f.). Descripción. https:// www.conicet.gov.ar/Conicet-descripcion/

Dancette, J. (1995). Parcours de traduction. Presses Universitaires de Lille.

Delisle, J. (1984). L'analyse du discours comme méthode de traduction. Ed. de 1'Université d'Ottawa.

Delisle, J. (2005). Le sens à travers l'histoire de la traduction, de l'Antiquité à la fin du xixe siècle. En F. Israël y M. Lederer (Eds.), La théorie interprétative de la traduction. Lettres modernes Minard. https://www.academia.edu/5995238/ Le_sens_\%C3\%A0_travers_lhistoire_de_la_ traduction_de_1Antiquit\%C3\%A9_\%C3\%A0_ la_fin_du_XIxe_si\%C3\%A8cl 
East, S., Laurence, T. y López, E. (s. f.). covID-19 y la situación de las trabajadoras de la salud en Argentina. Informe técnico. Organización Internacional del Trabajo, JNFPA, ONU Mujeres. https://argentina.unfpa.org/sites/default/files/pub-pdf/ wcms_754614.pdf

El Miri, M. y Mercier, D. (2018). «L'encampement» des réfugiés syriens au Moyen Orient : déborder les frontières de la vulnérabilité par le travail [Communication dans un congrès]. Les réfugiés syriens au Moyen Orient. Encampement, vulnérabilités et travail., Séminaire MIGTRAV; Séminaire MIMED; ANR LAJEH, Feb 2018, Aix-en-Provence, France. https:// hal.archives-ouvertes.fr/halshs-02135754

Facultad de Humanidades y Ciencias de la Educación (FaHCE), Universidad Nacional de La Plata (UNLP) (2016). Traductorado Público Nacional en Lengua Francesa. http://www.fahce. unlp.edu.ar/academica/areas/lenguas-y-literaturas-modernas/carreras/traductorado-en-frances

Freyre, M. L., Alliaud, A. G., Cagnolati, B. E., Forte Mármol, A., Gentile, A. M., Urrutia, M. I. (2001). Traducción científico-técnica: criterios de evaluación. Cuadernos de Lenguas Modernas, (3), 96-115. http://sedici.unlp.edu. ar/handle/10915/13027

Gamero Pérez, S. (2001). La traducción de textos técnicos. Ariel.

Gile, D. (1986). La compréhension des énoncés spécialisés chez le traducteur: quelques réflexions. Meta, 31(4), 363-360. http://dx.doi. org/10.7202/002906ar

Gouadec, D. (1989). Le traducteur, la traduction et l'entreprise. Afnor.

Hurtado Albir, A. (1996). La enseñanza de la traducción directa "general". Objetivos de aprendizaje y metodología. En A. Hurtado Albir (Ed.), La enseñanza de la traducción. Publicacions de la Universitat Jaume I, D. L.

Hurtado Albir, A. (2013). Traducción y traductología (6. ${ }^{a}$ ed.). Cátedra.

Institut National de la Statistique et des Études Économiques (INSEE) (2017). Nomenclatures des professions et catégories socioprofessionnelles. https:// www.insee.fr/fr/information/2406153
Instituto Argentino de Normalización y Certificación (IRAM) (2019). Norma IRAM 17100. Servicios de traducción. Requisitos para los servicios de traducción. https://iram.org.ar

Instituto Nacional de Estadística y Censos (2011). Encuesta permanente de hogares. Conceptos de Condición de actividad, subocupación horaria $y$ categoría ocupacional. https://www.indec.gob. $\mathrm{ar} / \mathrm{ftp} / \mathrm{cuadros} / \mathrm{menusuperior/eph/EPH}$ Conceptos.pdf

Laboratoire d'Économie et de Sociologie du Travail (LEST) (s. f.). https://www.lest.cnrs.fr/

Larose, R. (1989). Théories contemporaines de la traduction. PUQ.

Leclerc-Olive, M. (2016). Traduire les sciences humaines. Auteur, traducteur et incertitudes. Meta, 61(1), 42-59. https://doi. org/10.7202/1036982ar

Lederer, M. (2003). De l'interdépendance de la théorie et de la pratique en traduction. Revue de la SAFESU, 21(26), 13-21.

Malinowski Rubio, M. P. (2003). La lengua especializada en la traducción de textos literarios [Ponencia en congreso]. Actas del I Congreso Internacional de la Asociación Ibérica de Estudios de Traducción e Interpretación (AIETI) (Vol. 2). Granada, España. http://www.aieti.eu/wp-content/ uploads/AIETI_1_MPMR_Lengua.pdf

Marín, O. E. y Aguilar, M. C. (2009). Aproximación a una didáctica de la traducción de ciencias sociales y humanas. Mutatis Mutandis, Revista Latinoamericana de Traducción, 2(1), 98109. https://revistas.udea.edu.co/index.php/ mutatismutandis/article/view/1965/1664

Narvaja de Arnoux, E., Distéfano, M. y Pereira, C. (2002). La lectura y escritura en la universidad. Eudeba.

Neffa, J. C. (2015). Los riesgos psicosociales en el trabajo: contribución a su estudio. Centro de Estudios e Investigaciones Laborales - CEIL-CoNICET. http://www.ceil-CONICET.gov.ar/ wp-content/uploads/2015/11/Neffa-Riesgos-psicosociales-trabajo.pdf

Nord, C. (2003). El análisis contrastivo y cultural en la clase de lengua. Quaderns. Revista de traducció, (10), 23-39. https://ddd.uab.cat/record/2610 
Price, J. (2010 [2008]). La traducción de las ciencias sociales. Utopismos bueno y malo confrontados (S. T. Benítez, Trad.). Mutatis Mutandis. Revista Latinoamericana de Traducción, 3(1), 152-173. https://revistas.udea. edu.co/index.php/mutatismutandis/article/ view/6258/5770

Real Academia Española (2020). Diccionario de la lengua española (24. ${ }^{\circ}$ edición del tricentenario). https://dle.rae.es

Saint-Exupéry, A. (1931). Vol de nuit. Gallimard.

Sánchez Upegui, A. A. (2012). Análisis lingüístico de artículos de investigación en ciencias sociales y humanas. Lingüistica y Literatura, (62), 105-121. http://www.redalyc.org/articulo. oa? id $=476548730008$
Sapiro, G. (2012). La circulation des sciences humaines et sociales en traduction : enjeux et obstacles à l'heure de la globalisation. Traduire, (227), 5-15. https://doi.org/10.4000/ traduire. 465

Spoto Zabala, D. (2020). La evaluación de traducciones científico-técnicas en la formación de traductores y traductoras. Synergies Argentine, (6), 105-121. https://gerflint.fr/Base/Argentine6/spoto_zabala.pdf

Toury, G. (2004). Los estudios descriptivos de traducción y más allá. Metodología de la investigación en estudios de traducción (R. Rabadán y R. Merino, Trads.). Cátedra.

Van Dijk, T. (1983). La ciencia del texto. Un enfoque interdisciplinario. Paidós.

Cómo citar este artículo: Cagnolati, B. E. (2021). Formación de traductores universitarios: experiencias interdisciplinarias en el campo de la traducción de las ciencias sociales y humanas. $\mathrm{Mu}$ tatis Mutandis. Revista Latinoamericana de Traducción, 14(2), 642-661. https://doi.org/10.17533/ udea.mut.v14n2a17 